\title{
Effects of the posterior cruciate ligament and tibia insert thickness on tibiofemoral joint pressure in total knee arthroplasty: a cadaveric study
}

\author{
Xing Xin ${ }^{1,2}$, Hong Cai ${ }^{1}$, Zhongqiang Chen ${ }^{1,2}$ \\ ${ }^{1}$ Department of Orthopedics, Peking University Third Hospital, Beijing, China; ${ }^{2}$ Department of Orthopedics, Peking University International \\ Hospital, Beijing, China \\ Contributions: (I) Conception and design: All authors; (II) Administrative support: H Cai, Z Chen; (III) Provision of study materials: H Cai; (IV) \\ Collection and assembly of data: H Cai, X Xin; (V) Data analysis and interpretation: All authors; (VI) Manuscript writing: All authors; (VII) Final \\ approval of manuscript: All authors. \\ Correspondence to: Hong Cai. Department of Orthopedics, Peking University Third Hospital, No. 49 North Garden Road, Haidian District, Beijing \\ 100191, China. Email: hongcai@bjmu.edu.cn.
}

Background: Emerging knowledge has highlighted the significant role of the posterior cruciate ligament (PCL) in total knee arthroplasty (TKA). However, few studies have reported how the PCL affects tibiofemoral joint pressure (TFP), and differences in the effects of the PCL and the effects of tibial insert thickness on TFP remain unknown. Thus, this study used the sensor technique to analyze and compare the effects of PCL and tibial insert thickness on TFP during knee flexion.

Methods: Cruciate-retaining total knee arthroplasty (CR-TKA) was performed in 8 cadaveric knees. The PCL was partially released and completely resected sequentially while the tibial insert thickness was increased from 10- to $12-\mathrm{mm}$ at $1-\mathrm{mm}$ intervals. With the optimal tibial insert in place, the effects of PCL release and resection on medial and lateral TFP during knee flexion were analyzed. Medial tibiofemoral joint pressure (MTFP) with PCL retention and a 12-mm tibial insert was set as the baseline. The effects of PCL resection without tibial insert thickness reduction on MTFP were compared to the effects of PCL retention with a 1- or 2-mm thinning of the tibial insert on MTFP during knee flexion.

Results: PCL resection significantly reduced MTFP at $90^{\circ}$ and $120^{\circ}$ of knee flexion $(\mathrm{P}=0.01$ and $\mathrm{P}=0.03$, respectively). Partial release and complete resection of the PCL did not significantly reduce lateral tibiofemoral joint pressure (LTFP) at $10^{\circ}, 30^{\circ}, 60^{\circ}, 90^{\circ}$ and $120^{\circ}$ of knee flexion $(\mathrm{P}=0.68, \mathrm{P}=0.60, \mathrm{P}=0.62$, $\mathrm{P}=0.21$ and $\mathrm{P}=0.12$, respectively). At $10^{\circ}, 30^{\circ}$, and $60^{\circ}$ of knee flexion, a $1-\mathrm{mm}$ reduction in the tibial insert had a greater effect on MTFP than did the resection of the PCL. In contrast, at $90^{\circ}$ of knee flexion, MTFP was more affected by PCL resection than by a $2-\mathrm{mm}$ reduction of the tibial insert.

Conclusions: The PCL predominantly affects MTFP at $90^{\circ}$ and $120^{\circ}$ of knee flexion. The impact of PCL resection on $\mathrm{MTFP}$ at $90^{\circ}$ flexion was higher than the impact of a $2-\mathrm{mm}$ thinning of the tibial insert.

Keywords: Posterior cruciate ligament (PCL); tibiofemoral joint pressure (TFP); total knee arthroplasty (TKA)

Submitted Oct 15, 2021. Accepted for publication Jan 14, 2022.

doi: 10.21037/atm-21-5487

View this article at: https://dx.doi.org/10.21037/atm-21-5487 


\section{Introduction}

The posterior cruciate ligament (PCL) plays a very important role in knee kinematics, functional recovery, and prosthesis survival after PCL-retaining total knee arthroplasty (CR-TKA). An intact or well-preserved PCL in TKA can effectively reproduce physiological kinematics such as femur rollback (1), lateral pivot at early flexion, and medial pivot at mild flexion (2). Furthermore, a wellpreserved PCL can help restore daily movement, such as walking and climbing stairs, after TKA (3). However, there is no consensus regarding the effects of the PCL on softtissue balance in the knee $(2,4-11)$. While some studies have reported that PCL release selectively increases the flexion gap more than the extension gap and accordingly causes a mismatch of the extension and flexion gaps, other studies have not confirmed these results $(2,10)$. In addition, some studies have reported that the lateral flexion gap increases more significantly than does the medial gap after PCL resection $(8,11)$. Other studies have not confirmed this $(4,9)$.

In the above studies, the effects of the PCL on knee balance were analyzed by applying certain force to the knee in extension or flexion. The results of these studies were influenced by various elements such as the experience of the surgeons $(12,13)$, soft tissue stiffness, and joint distraction force $(14,15)$. Compared with joint space extension by a set distraction force, the tibiofemoral joint pressure (TFP) is a more objective and accurate means to reflect knee balance (16). However, few studies have reported how the PCL affects intraarticular pressure, and differences in the effects of the PCL and the effects of tibial insert thickness on TFP remain unknown. Therefore, it was usually a difficult choice between ligament resection and tibia insert reduction when dealing with a high joint pressure in the CR-TKA.

In this study, we used a pressure-sensing tibial insert, which provided objective, real-time quantitative feedback on joint pressure, to sequentially analyze the effects of PCL release and resection on TFP during knee flexion. We then compared the effects of PCL resection and tibial insert thickness reduction on medial TFP (MTFP) during knee flexion. We present the following article in accordance with the MDAR reporting checklist (available at https://atm. amegroups.com/article/view/10.21037/atm-21-5487/rc).

\section{Methods}

\section{Specimen preparation}

Eight fresh-frozen cadaveric knees were obtained for this study (4 right and 4 left knees). The specimen characteristics were as follows: mean age, 70.25 (SD 4.9) years; age range, 64 to 78 years; mean weight, 66.25 (SD 5.9) $\mathrm{kg}$; and mean height, 158.5 (SD 4.5) cm. No trauma or surgical history was present in the knee joints.

\section{Measurement device}

A goniometer (i-Join, Shanghai, China) was used in this study to record knee flexion angles. This device used a 3 -axis accelerometer [a programmable force sensing resistor (FSR) $\pm 2, \pm 4, \pm 8, \pm 16 \mathrm{~g}$ ] and a 3 -axis gyroscope (sensitivity error $\pm 1 \%$ and noise \pm 4 mdps $/ \sqrt{ } \mathrm{Hz}$ ) for data collection. The flexion angle was calculated by a specific algorithm. The angle measurement error was $\pm 1^{\circ}$. During TKA surgery, the goniometer was attached to the proximal thigh and distal calf, and the resulting angles were displayed on the terminal (Figure 1).

A pressure-sensing tibial insert (i-Join) able to record TFP at any flexion angle during TKA surgery was used in this study to measure MTFP and lateral TFP (LTFP) in the knee joints (Figure 2). This device integrates a pressure sensor with a tibial insert. The pressure sensor is a 6-channel, 24-bit delta-sigma ADC that achieves exceptional performance while consuming very low power. Six pressure-sensing points were separately embedded in the medial and lateral sides of the tibial insert depending on tibiofemoral pressure distribution. These pressure-sensing points were able to record pressure at different points, directions, and angles with good sensitivity. Based on the data obtained from the sensors, the MTFP and LTFP were calculated using a specific algorithm. A third-party testing center used a push-pull force gauge to calibrate the pressure-sensing tibial insert. The measurement error of this device was $\pm 10 \mathrm{~N}$.

\section{Surgical procedure}

The cadaveric specimens, intact from head to toe, were stored at $-20{ }^{\circ} \mathrm{C}$ and thawed overnight at room temperature 

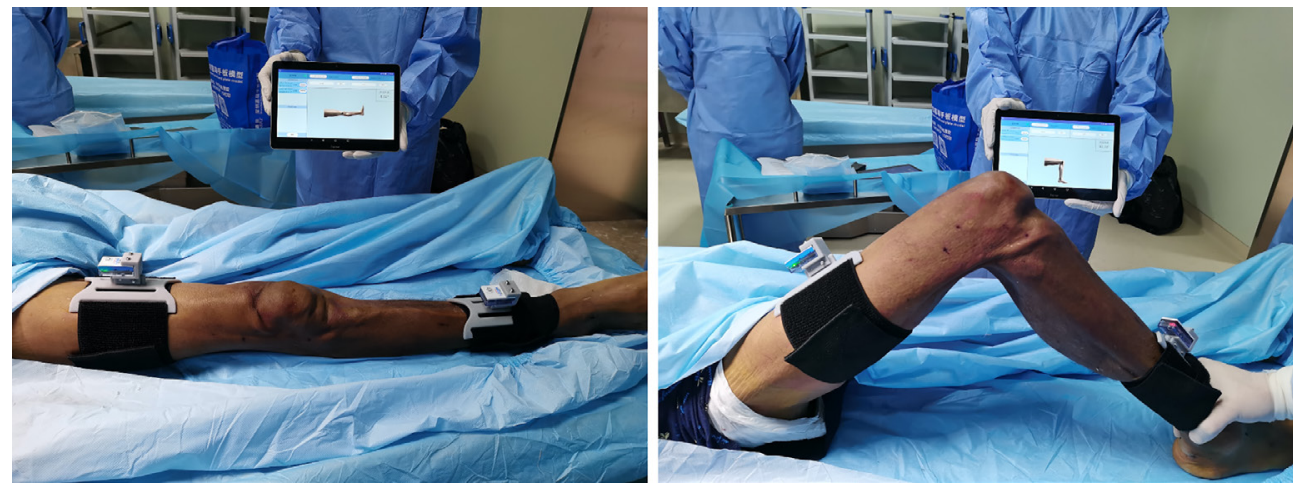

Figure 1 The goniometer for recording the range of motion in the knee joints. The 2 components are attached to the proximal thigh and the distal calf.

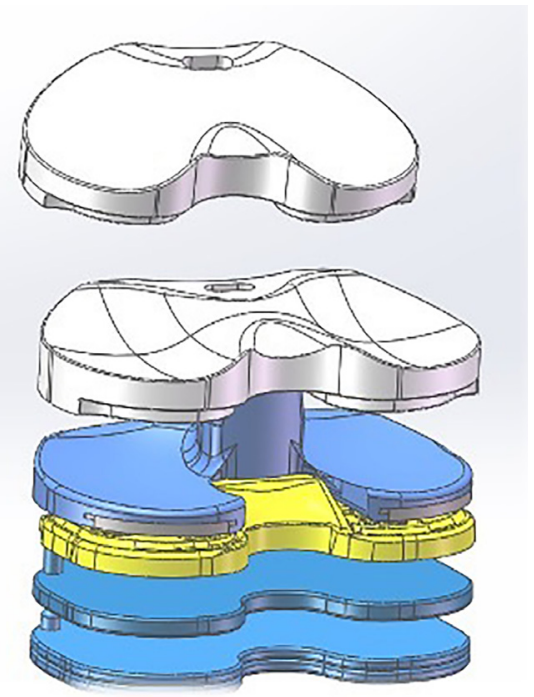

Figure 2 The pressure-sensing tibial insert for measuring medial and lateral tibiofemoral joint pressure.

before the TKA surgery. The surgery was performed on a surgical table with knee and hip joints free to move and the heel braced by a bolster. A CR-TKA prosthesis (A3GT, Ak Medical, Beijing, China) was implanted in each knee joint, following the instructions of the manufacturer and using the measured resection technique. First, the knee joint was exposed by the standard medial parapatellar approach. Then the distal femur was cut at the valgus at $6^{\circ}$ to the femoral anatomical axis. The PCL and tibial attachment site were preserved with an osteotome before the tibial osteotomy. Finally, the proximal tibia was cut perpendicular to the tibial anatomical axis with a $3^{\circ}$ posterior inclination. No bone defects of the tibial plateau were found in the knee joints.
The rotation of the femoral prostheses was determined by the femoral epicondylar axis and Whiteside's line. These operations were primarily performed by a senior surgeon (Hong Cai) who performs over 300 knee arthroplasties a year.

\section{Measurement of joint pressure}

With the tibial and femoral prostheses in place and the PCL intact, a pressure-sensing tibial insert (i-Join) with an initial thickness of $12 \mathrm{~mm}$ was inserted to measure MTFP and the LTFP at $10^{\circ}, 30^{\circ}, 60^{\circ}, 90^{\circ}$, and $120^{\circ}$ of knee flexion as determined by the goniometer. The total TFP (TP) was calculated as the sum of MTFP and LTFP, and TFP distribution (PD) was calculated as the ratio of MTFP to TP. The insert thickness was gradually increased from $10-$ to $12-\mathrm{mm}$ at $1-\mathrm{mm}$ intervals. The optimal tibial insert was determined according to knee balance criteria, which was defined as MTFP of $222.5 \mathrm{~N}_{ \pm} 88.9 \mathrm{~N}$ and LTFP of $155.8 \mathrm{~N} \pm 89 \mathrm{~N}$, with a difference between the MTFP and LTFP of $66.8 \mathrm{~N} \pm 22.3 \mathrm{~N}$ (17). A \#11 surgical scalpel blade was used to sequentially release $50 \%$ (the anterolateral bundle) of the PCL and $100 \%$ of the PCL at the femoral attachment site (Figure 3). With the optimal tibial insert in place, the effects of PCL release and resection on MTFP, LTFP, TP, and PD during knee flexion were then evaluated.

Due to the different tensions in the medial and lateral soft tissues of the knee joints, MTFP and LFTP do not increase equally with the increased thickness of the tibial insert. In fact, an increase in insert thickness may lead to the reduction of MTFP or LTFP due to the "seesaw" effect. For a normal knee joint, medial tissue tension is reportedly higher than lateral tissue tension. Therefore, 

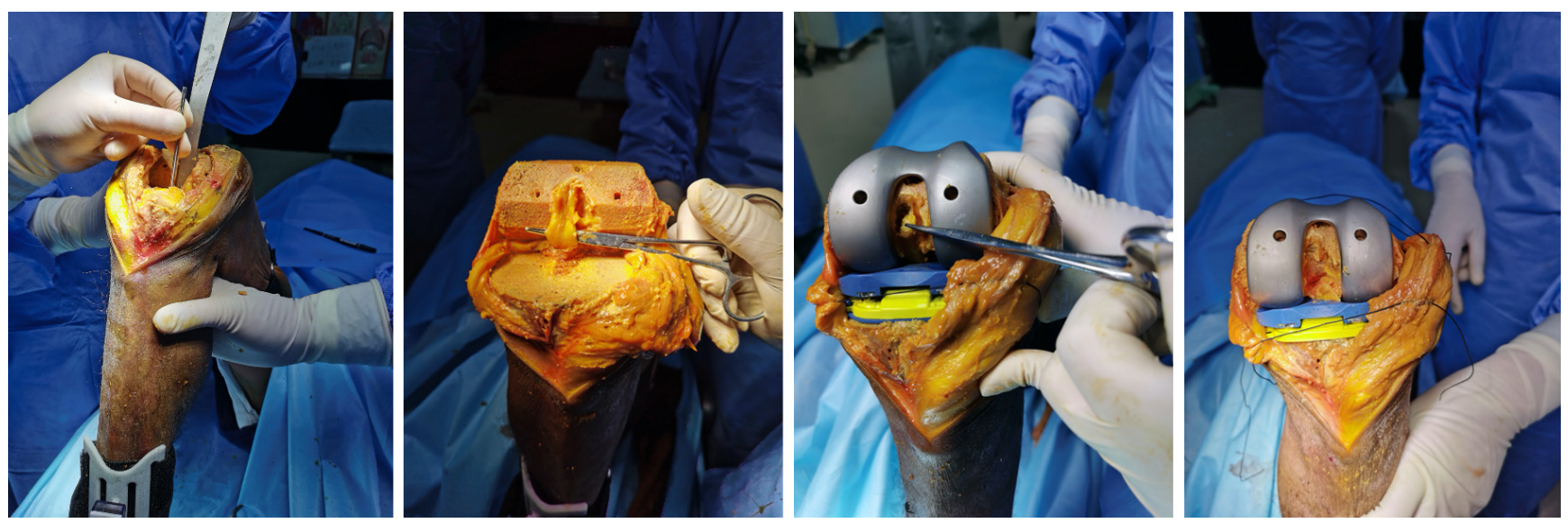

Figure 3 The posterior cruciate ligament (PCL) was partially released and completely resected at the femoral site.

we chose to analyze the effects of PCL resection and insert thickness on MTFP. Knee joints that were balanced with PCL retention and a $10-\mathrm{mm}$ tibial insert were included in the following analysis. MTFP with PCL retention and a $12-\mathrm{mm}$ tibial insert was set as the baseline. The changes of insert thickness from $12-\mathrm{mm}$ to $11-\mathrm{mm}$ and from $12-\mathrm{mm}$ to $10-\mathrm{mm}$ was respectively called $1-\mathrm{mm}$ and $2-\mathrm{mm}$ thinning of tibial insert. We compared the effects on MTFP of PCL resection without tibial insert thickness reduction and PCL retention with a 1 - or 2 -mm thinning of the tibial insert during knee flexion.

\section{Ethical statement}

The study was conducted in accordance with the Declaration of Helsinki (as revised in 2013). This cadaveric study was performed at a dedicated institution that was not at our institution. We had permission for conducting this cadaveric study. All the cadavers were donated voluntarily, and the donors expressed their willingness to use them for further research.

\section{Statistical analysis}

The normality of the continuous variables was analyzed by a Shapiro-Wilk test. Joint pressure was measured 3 times, and the intraclass correlation coefficient (ICC) was used for reliability analysis. The differences in TP, MTFP, LTFP, and PD with PCL release and resection at different degrees of knee flexion were analyzed using 1-way analysis of variance (ANOVA) tests and Bonferroni post hoc tests. All values are expressed as mean \pm SD. Statistical analyses were performed with the SPSS 19.0 software for Windows (IBM Corp., Armonk, NY, USA). A 2-tailed P value of 0.05 was considered statistically significant.

\section{Results}

\section{Characteristics of cadaveric specimens}

The specimens had a mean age of 70 years, mean weight of $66 \mathrm{~kg}$, and mean height of $159 \mathrm{~cm}$. Only mild degeneration of articular cartilage was found in the knee joints. The optimal thicknesses of the tibial inserts for each knee joint are shown in Table 1.

\section{Reliability analysis of the pressure-sensing tibial insert}

The ICC for the pressure-sensing tibial insert is shown in Table 2. The device showed good consistency in measuring joint pressure with different PCL treatments and different tibial insert thicknesses $(\mathrm{P}<0.001)$.

\section{Effects of the PCL on TFP}

Data were normally distributed, and changes in MTFP, LTFP, TP, and PD during knee flexion after PCL release and resection are shown in Table 3.

MTFP decreased from $10^{\circ}$ to $60^{\circ}$ of knee flexion and then gradually increased from $60^{\circ}$ to $120^{\circ}$ of knee flexion. LTFP decreased slightly from $10^{\circ}$ to $120^{\circ}$ of knee flexion. When the PCL was $50 \%$ and $100 \%$ resected, MTFP did not significantly change at $10^{\circ}, 30^{\circ}$ and $60^{\circ}$ of knee flexion $(\mathrm{P}=0.71, \mathrm{P}=0.49$, and $\mathrm{P}=0.30)$. A $50 \% \mathrm{PCL}$ resection did not significantly change MTFP at $90^{\circ}$ and $120^{\circ}$ of knee 
Table 1 Characteristics of cadaveric specimen

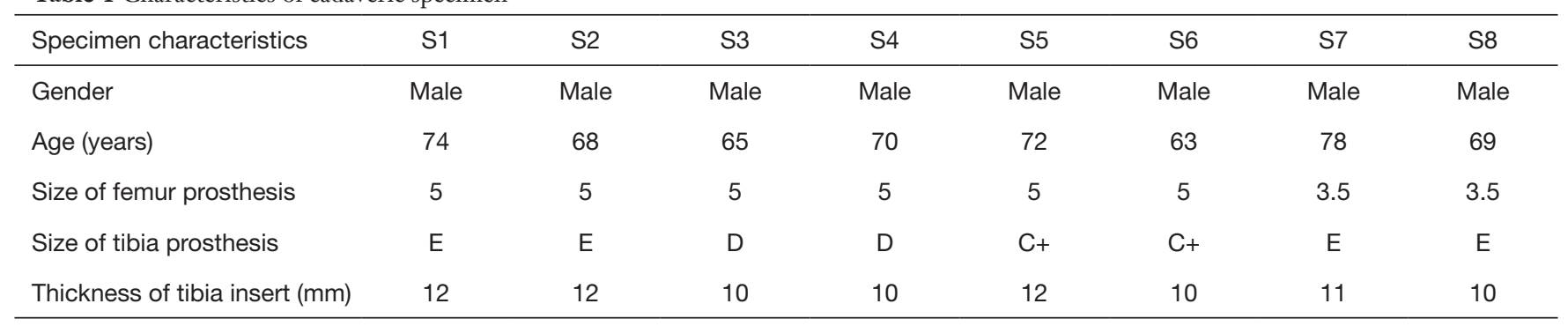

Table 2 The intraclass correlation coefficient for pressure sensor tibia insert ( $95 \%$ confidence interval)

\begin{tabular}{|c|c|c|c|c|c|c|}
\hline Variable & \multicolumn{2}{|c|}{ PCL-retention } & \multicolumn{2}{|c|}{ PCL-release } & \multicolumn{2}{|c|}{ PCL-resection } \\
\hline \multicolumn{7}{|l|}{$10 \mathrm{~mm}$} \\
\hline MTFP & $0.976(0.960,0.987)$ & 0.000 & $0.984(0.971,0.991)$ & 0.000 & $0.934(0.884,0.964)$ & 0.000 \\
\hline LTFP & $0.978(0.963,0.988)$ & 0.000 & $0.975(0.956,0.987)$ & 0.000 & $0.959(0.928,0.978)$ & 0.000 \\
\hline \multicolumn{7}{|l|}{$11 \mathrm{~mm}$} \\
\hline MTFP & $0.975(0.958,0.986)$ & 0.000 & $0.989(0.980,0.994)$ & 0.000 & $0.931(0.877,0.963)$ & 0.000 \\
\hline LTFP & $0.980(0.965,0.989)$ & 0.000 & $0.991(0.984,0.995)$ & 0.000 & $0.893(0.812,0.942)$ & 0.000 \\
\hline \multicolumn{7}{|l|}{$12 \mathrm{~mm}$} \\
\hline MTFP & $0.966(0.943,0.981)$ & 0.000 & $0.974(0.953,0.987)$ & 0.000 & $0.952(0.906,0.974)$ & 0.000 \\
\hline
\end{tabular}

MTFP, medial tibiofemoral joint pressure; LTFP, lateral tibiofemoral joint pressure; PCL, posterior cruciate ligament; ICC, intraclass correlation coefficient; $\mathrm{Cl}$, confidence interval.

flexion ( $\mathrm{P}=0.19$ and $\mathrm{P}=0.66$, respectively). The total removal of the PCL significantly reduced MTFP at $90^{\circ}$ and $120^{\circ}$ of knee flexion $(\mathrm{P}=0.01$ and $\mathrm{P}=0.03$, respectively). LTFP did not significantly change at $10^{\circ}, 30^{\circ}, 60^{\circ}, 90^{\circ}$ and $120^{\circ}$ of knee flexion after $50 \%$ and $100 \%$ of PCL resection $(\mathrm{P}=0.68$, $\mathrm{P}=0.60, \mathrm{P}=0.62, \mathrm{P}=0.21$, and $\mathrm{P}=0.12 ;$ Figure $4 A, 4 B)$.

The TP decreased from $10^{\circ}$ to $60^{\circ}$ of knee flexion and then gradually increased from $60^{\circ}$ to $120^{\circ}$ degrees of knee flexion. When the PCL was $50 \%$ and $100 \%$ resected, the TP did not change significantly at $10^{\circ}, 30^{\circ}$ and $60^{\circ}$ of knee flexion $(\mathrm{P}=0.50, \mathrm{P}=0.16$, and $\mathrm{P}=0.06)$. A $50 \%$ PCL resection did not significantly change the TP at $90^{\circ}$ and $120^{\circ}$ of knee flexion $(\mathrm{P}=0.12$ and $\mathrm{P}=0.51$, respectively). The total removal of the PCL significantly reduced the TP at $90^{\circ}$ and $120^{\circ}$ of knee flexion $(\mathrm{P}=0.003$ and $\mathrm{P}=0.01$, respectively; Figure $4 C)$.

PD gradually increased from $10^{\circ}$ to $90^{\circ}$ of knee flexion but then changed little from $90^{\circ}$ to $120^{\circ}$ of knee flexion.
After the partial and complete resection of the PCL, PD did not change significantly at $10^{\circ}, 30^{\circ}, 60^{\circ}, 90^{\circ}$ and $120^{\circ}$ of knee flexion $(\mathrm{P}=0.96, \mathrm{P}=0.93, \mathrm{P}=0.91, \mathrm{P}=0.60$, and $\mathrm{P}=0.64$; Figure 4D).

\section{Effects of tibial insert thinning and PCL resection on joint pressure}

The optimal thickness of the tibial insert was found to be $10 \mathrm{~mm}$ in 5 of 8 knee joints. In 4 of those 5 knee joints, MTFP primarily increased as the thickness of the tibial insert increased. In these 4 knee joints, MTFP with a $12-\mathrm{mm}$ tibial insert and PCL retention was used as the baseline. The changes of insert thickness from 12- to $11-\mathrm{mm}$ and from 12 - to $10-\mathrm{mm}$ was respectively called $1-$ and 2-mm thinning of tibial insert. We compared the effects on MTFP of PCL resection without tibial insert thickness 
reduction and PCL retention with a 1- or $2-\mathrm{mm}$ thinning of the tibial insert during knee flexion. At $10^{\circ}, 30^{\circ}$ and $60^{\circ}$ of knee flexion, the decrease of MTFP caused by $1-\mathrm{mm}$ thinning of the tibial insert without PCL resection was higher than the decrease of MTFP caused by PCL resection without tibial insert reduction (Table 4). However, at $90^{\circ}$ of knee flexion, the reduction in MTFP caused by the PCL resection without tibial insert reduction was higher than the reduction in MTFP caused by a $2-\mathrm{mm}$ thinning of the tibial insert without PCL resection (Figure 5).

\section{Discussion}

The PCL plays an essential role in knee kinematics, functional recovery, and prosthesis survival after CRTKA. Still, its effect on the MTFP and LTFP of the knee joint remains unknown. In this cadaveric study, we used the sensor technique to sequentially quantify the effects of PCL release and resection on TFP during knee flexion. We also compared the effects of PCL resection and tibial insert thinning on MTFP. One important finding was that PCL resection significantly decreased the TP and predominantly decreased MTFP at $90^{\circ}$ and $120^{\circ}$ of knee flexion. Another finding was that the PCL did not affect PD in the knee joint during knee flexion. We also found that PCL resection had a greater impact on MTFP than did a 2-mm reduction of the tibial insert at $90^{\circ}$ of knee flexion.

Several papers have reported the effects of the PCL on knee balance. Matthews et al. (1) used a navigated knee system to quantify the impact of PCL on joint gaps in 10 cadaveric knee joints. They conducted 6 sequential testing regimens with the knee intact, a cruciate-retaining prosthesis, and a posterior stabilized prosthesis in place. Their results suggested that the PCL did not affect tibiofemoral joint gaps during knee flexion. In a casecontrol study of 41 patients with varus knee osteoarthritis (OA) by Oshima et al. (2), tibiofemoral joint gaps at knee extension and flexion did not increase significantly when the PCL was resected without osteotomy or collateral ligament release. Contrary to the above results, Schnurr et al. (18) carried out a retrospective study of 50 patients with varus knee OA and found that PCL resection predominantly increased the medial gap at $90^{\circ}$ of knee flexion. However, in a study of 45 osteoarthritic patients by Yagishita et al. (19), the lateral joint gap at $90^{\circ}$ of knee flexion increased more than did the medial one after PCL resection. Kayani et al. (8) performed a prospective study of 110 patients to find that PCL resection significantly increased the height of 
A

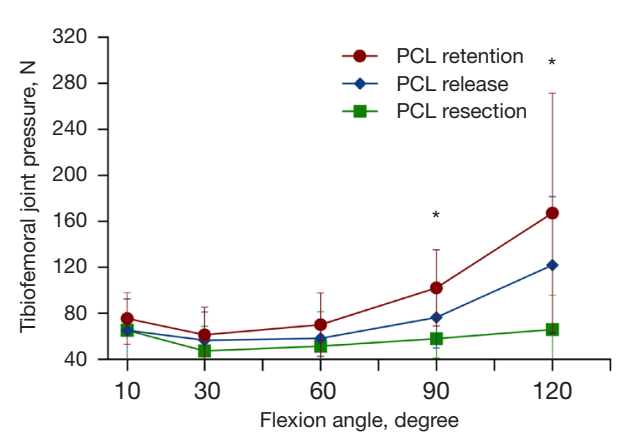

Effects of PCL on the medial tibiofemoral joint pressure during knee flexion

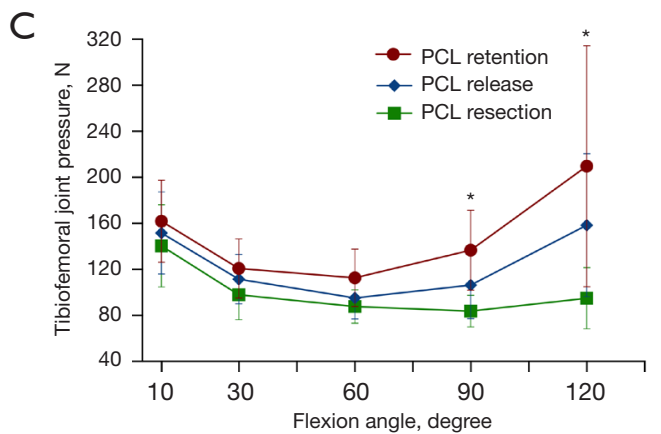

Effects of PCL on the total tibiofemoral joint pressure during knee flexion
B

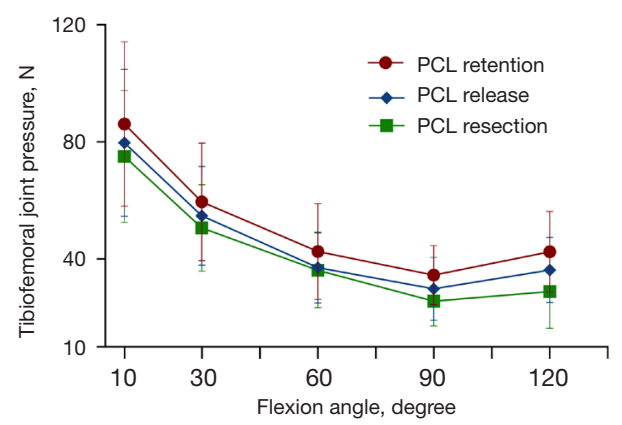

Effects of PCL on the lateral tibiofemoral joint pressure during knee flexion

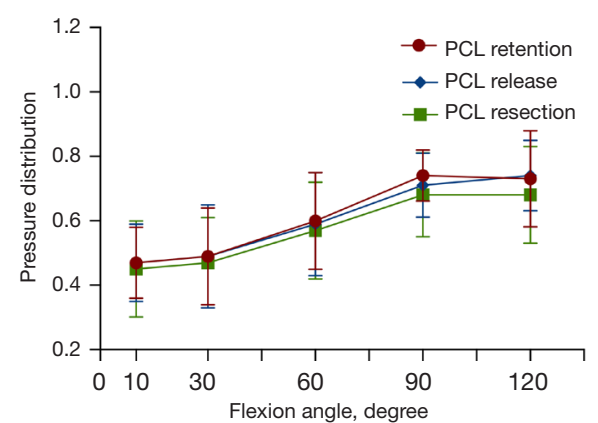

Effects of PCL on pressure distribution of tibiofemoral joint during knee flexion

Figure 4 Effects of posterior cruciate ligament (PCL) release and resection on the medial tibiofemoral joint pressure (A), lateral tibiofemoral joint pressure (B), total tibiofemoral joint pressure (C), and pressure distribution of tibiofemoral joint (D). The PCL resection significantly reduced the medial tibiofemoral joint pressure and total tibiofemoral joint pressure at $90^{\circ}$ and $120^{\circ}$ of knee flexion. ${ }^{*}, \mathrm{P}<0.05$.

Table 4 Effects of PCL resection and tibia insert reduction on the tibiofemoral joint pressure

\begin{tabular}{lccc}
\hline ROM (deg) & PCL resection* & Thickness minus 1 mm & Thickness minus 2 mm $^{\#}$ \\
\hline 10 & $28.58 \pm 23.50$ & $38.26 \pm 18.54$ & $51.51 \pm 70.58$ \\
30 & $38.90 \pm 20.80$ & $50.54 \pm 23.94$ & $57.50 \pm 64.02$ \\
60 & $52.06 \pm 24.88$ & $61.39 \pm 40.89$ & $73.20 \pm 56.78$ \\
90 & $99.40 \pm 33.74$ & $58.59 \pm 43.40$ & $84.68 \pm 66.09$ \\
\hline
\end{tabular}

*, the reduction of the medial tibiofemoral joint pressure with 12-mm tibia insert after the PCL resection; ", the reduction of the medial tibiofemoral joint pressure with the intact PCL after the 1-mm or 2-mm reduction in the 12-mm tibia insert. PCL, posterior cruciate ligament; ROM, range of motion.

the lateral compartment significantly more than that of the medial compartment at $90^{\circ}$ of knee flexion.

Several factors might have contributed to the above discrepancy. The first potential factor is the extensor mechanism (patella eversion or reduction). Yoshino et al. (20) used a tensor system to quantify the impact of patella eversion and reduction on joint gaps in posteriorcruciate substituting TKA (PS-TKA) and CR-TKA. The results showed that patellar condition had a significant effect on the joint space at $90^{\circ}$ flexion only in PS-TKA. In a study by Sculco et al. (21), the LTFP increased significantly during knee flexion as the patella was laterally everted in PS-TKA. To avoid the influence of the knee extensor mechanism, joint pressure was measured with a patella reduction in TKA. A second factor is that the effects of the PCL on joint space and pressure depend on the condition of the medial 


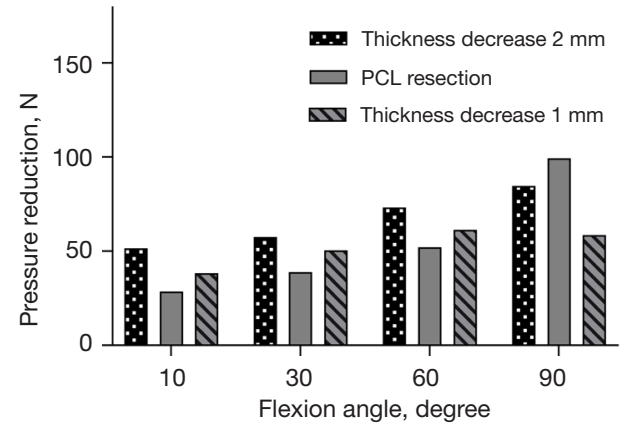

The effects of thickness reduction and PCL resection on joint pressure

Figure 5 Effects of posterior cruciate ligament (PCL) resection and tibial insert thickness reduction on medial tibiofemoral joint pressure (MTFP).

collateral ligament (MCL). Kim et al. (22) reported that MCL release significantly extended the medial tibiofemoral joint gap in PS-TKA, but not in CR-TKA. This suggested that the PCL and MCL jointly determine the tension of the medial compartment of the knee joint. In the study of Oshima et al., PCL resection with the MCL intact did not produce significant increases in joint gaps during knee flexion (2). A third influencing factor is the degree of PCL degeneration. The stiffness of a severely degenerated PCL is significantly greater than that of a mildly degenerated one (23). Therefore, removing PCLs with different degrees of degeneration may produce completely different effects on joint space or pressure. However, little has been reported on this hypothesis. Finally, the sequence of soft tissue release also affects the results. MCL release followed by PCL resection significantly increased the medial tibiofemoral joint gap of knee flexion (18). PCL resection with the MCL intact significantly increased the lateral tibiofemoral joint gap of knee flexion $(8,11,19)$. The reason for this was that the tension of the lateral knee joints was lower than that of the medial structures during knee flexion in both normal people and OA patients (24-27). Therefore, the lateral compartment expansion was larger than the medial one. The MCL has been found to play a more significant role than the PCL in medial knee joint stiffness at $90^{\circ}$ of knee flexion (22). PCL resection without MCL release cannot significantly reduce the medial tension of the knee joints. Therefore, the lateral compartment still opened more than the medial when the tibiofemoral joint was distracted $(8,11)$. On the other hand, PCL resection following MCL release significantly reduced the medial tension during knee flexion (18). Therefore, the medial compartment opened more than the lateral when the tibiofemoral joint was distracted.

A major finding of our study was the predominant effect of the PCL on joint pressure during knee flexion, which was consistent with previous studies (18). However, our study found that the partial release and removal of the PCL did not significantly increase the ratio of medial to lateral compartment pressure, which was inconsistent with previous results (28). One possible reason for this may be that none of the cadavers in this study showed end-stage knee OA. Previous studies have revealed the contracture of medial soft tissue, particularly the MCL, to be frequently present in advanced knee OA, and the MCL to have a larger effect than the PCL on medial soft tissue tension (22). However, the tension of MCL in normal knee joint was lower than that in OA knee joint. The less rigid MCL and PCL resection in normal knee joint may an equal contribution to medial and lateral joint pressure. In addition, PCL resection decreased joint pressure more than did a reduction in the tibial insert thickness at $90^{\circ}$ of knee flexion. All these results show that the PCL significantly affects the medial pressure of the knee joint at $90^{\circ}$ of knee flexion.

Our study had some limitations. The knee specimens used in this study did not show severe OA. Whether these results are applicable to patients with advanced knee OA needs further study. However, previous studies have found that there is no difference in the stiffness of medial and lateral soft tissue between OA cadaveric knees, non-OA cadaveric knees, and OA knees. Therefore, it is reasonable to speculate that our conclusions may be relevant to OA knee joints. Furthermore, the PCL in OA knees is supposedly more rigid and shorter than in normal knee joints (29). However, this hypothesis has not yet been confirmed in high-quality studies. Despite the potential differences in PCL tension between OA and normal knee joints, the effects of the PCL on the joint space or pressure seem consistent. Forge et al. discovered that the PCL plays a predominant role in the medial flexion space in cadaveric knee joints without OA (9). This was also found to be true in OA knee joints. Tang et al. found that PCL resection significantly increased the medial to lateral compartment ratio (28). In addition, Yasushi et al. confirmed the major contribution of the PCL to the medial flexion gap in OA knee joints (2).

\section{Conclusions}

The PCL predominantly affects joint pressure of the medial compartment at $90^{\circ}$ of knee flexion. The effects of PCL 
resection on joint pressure were greater than the effects of 1 - and 2-mm reductions in tibial insert thickness at $90^{\circ}$ of knee flexion.

\section{Acknowledgments}

The authors are very grateful to the i-Join company (China) and Ak Medical (China) for providing the knee joint prostheses, the pressure-sensing tibial inserts, and the goniometer for this study. The authors would like to thank Dr. Yu Zhenguo, Mr. Chang Tao, Mr. Cai Zhen, Mr. Wu Weike, Mr. Li Guangyong, and Dr. Xiong Chenao for their assistance in the operations. The authors would also like to thank AES (https://editing.amegroups.cn) for English language editing.

Funding: None.

\section{Footnote}

Reporting Checklist: The authors have completed the MDAR reporting checklist. Available at https://atm.amegroups. com/article/view/10.21037/atm-21-5487/rc

Data Sharing Statement: Available at https://atm.amegroups. com/article/view/10.21037/atm-21-5487/dss

Peer Review File: Available at https://atm.amegroups.com/ article/view/10.21037/atm-21-5487/prf

Conflicts of Interest: All authors have completed the ICMJE uniform disclosure form (available at https://atm. amegroups.com/article/view/10.21037/atm-21-5487/coif). The authors have no conflicts of interest to declare.

Ethical Statement: The authors are accountable for all aspects of the work in ensuring that questions related to the accuracy or integrity of any part of the work are appropriately investigated and resolved. The study was conducted in accordance with the Declaration of Helsinki (as revised in 2013). This cadaveric study was performed at a dedicated institution that was not at our institution. We had permission for conducting this cadaveric study. All the cadavers were donated voluntarily, and the donors expressed their willingness to use them for further research.

Open Access Statement: This is an Open Access article distributed in accordance with the Creative Commons
Attribution-NonCommercial-NoDerivs 4.0 International License (CC BY-NC-ND 4.0), which permits the noncommercial replication and distribution of the article with the strict proviso that no changes or edits are made and the original work is properly cited (including links to both the formal publication through the relevant DOI and the license). See: https://creativecommons.org/licenses/by-nc-nd/4.0/.

\section{References}

1. Matthews J, Chong A, McQueen D, et al. Flexionextension gap in cruciate-retaining versus posteriorstabilized total knee arthroplasty: a cadaveric study. J Orthop Res 2014;32:627-32.

2. Oshima Y, Majima T, Iizawa N, et al. The Influence of Posterior Cruciate Ligament Resection on Tibiofemoral Joint Gap in Varus Osteoarthritic Knees. J Knee Surg 2022;35:323-30.

3. Andriacchi TP, Galante JO, Fermier RW. The influence of total knee-replacement design on walking and stairclimbing. J Bone Joint Surg Am 1982;64:1328-35.

4. Kadoya Y, Kobayashi A, Komatsu T, et al. Effects of posterior cruciate ligament resection on the tibiofemoral joint gap. Clin Orthop Relat Res 2001;(391):210-7.

5. Dorr LD, Boiardo RA. Technical considerations in total knee arthroplasty. Clin Orthop Relat Res 1986;(205):5-11.

6. Ma Y, Chen WJ, Nagamine R. Comparative evaluation of posterior cruciate ligament in total knee arthroplasty. J Orthop Surg (Hong Kong) 2017;25:2309499017690976.

7. Tsukeoka T, Tsuneizumi Y, Yoshino K. The rectangular flexion gap is associated with an increased knee flexion angle in a cruciate-sacrificing rotating platform mobile-bearing total knee arthroplasty. J Orthop Sci 2017;22:313-7.

8. Kayani B, Konan S, Horriat S, et al. Posterior cruciate ligament resection in total knee arthroplasty: the effect on flexion-extension gaps, mediolateral laxity, and fixed flexion deformity. Bone Joint J 2019;101-B:1230-7.

9. Foge DA, Baldini TH, Hellwinkel JE, et al. The Role of Complete Posterior Cruciate Ligament Release in Flexion Gap Balancing for Total Knee Arthroplasty. J Arthroplasty 2019;34:S361-5.

10. Burkhart TA, Perry KI, Dobbin E, et al. Effect of Soft Tissue Releases on Joint Space Opening in Total Knee Arthroplasty. J Arthroplasty 2016;31:2912-6.

11. Chaiyakit P, Meknavin S, Hongku N. Effects of posterior cruciate ligament resection in total knee arthroplasty using computer assisted surgery. J Med Assoc Thai 2009;92 
Suppl 6:S80-4.

12. Elmallah RK, Mistry JB, Cherian JJ, et al. Can We Really "Feel" a Balanced Total Knee Arthroplasty? J Arthroplasty 2016;31:102-5.

13. MacDessi SJ, Gharaibeh MA, Harris IA. How Accurately Can Soft Tissue Balance Be Determined in Total Knee Arthroplasty? J Arthroplasty 2019;34:290-294.e1.

14. Nagai K, Muratsu H, Matsumoto T, et al. Soft tissue balance changes depending on joint distraction force in total knee arthroplasty. J Arthroplasty 2014;29:520-4.

15. Asano H, Muneta T, Hoshino A. Stiffness of soft tissue complex in total knee arthroplasty. Knee Surg Sports Traumatol Arthrosc 2008;16:51-5.

16. Gustke KA, Golladay GJ, Roche MW, et al. A Targeted Approach to Ligament Balancing Using Kinetic Sensors. J Arthroplasty 2017;32:2127-32.

17. Risitano S, Karamian B, Indelli PF. Intraoperative loadsensing drives the level of constraint in primary total knee arthroplasty: Surgical technique and review of the literature. J Clin Orthop Trauma 2017;8:265-9.

18. Schnurr C, Eysel P, König DP. Is the effect of a posterior cruciate ligament resection in total knee arthroplasty predictable? Int Orthop 2012;36:83-8.

19. Yagishita K, Muneta T, Ikeda H. Step-by-step measurements of soft tissue balancing during total knee arthroplasty for patients with varus knees. J Arthroplasty 2003;18:313-20.

20. Yoshino N, Watanabe N, Fukuda Y, et al. The influence of patellar dislocation on the femoro-tibial loading during total knee arthroplasty. Knee Surg Sports Traumatol Arthrosc 2011;19:1817-22.

21. Sculco P, Gruskay J, Nodzo S, et al. The Role of the Tourniquet and Patella Position on the Compartmental Loads During Sensor-Assisted Total Knee Arthroplasty. J

Cite this article as: $\mathrm{Xin} \mathrm{X}$, Cai $\mathrm{H}$, Chen Z. Effects of the posterior cruciate ligament and tibia insert thickness on tibiofemoral joint pressure in total knee arthroplasty: a cadaveric study. Ann Transl Med 2022;10(8):437. doi: 10.21037/atm21-5487
Arthroplasty 2018;33:S121-5.

22. Kim SM, Jang SW, Seo JG, et al. Comparison of cruciate retaining and PCL sacrificing TKA with respect to medial and lateral gap differences in varus knees after medial release. J Arthroplasty 2015;30:26-30.

23. Yercan HS, Ait Si Selmi T, Sugun TS, et al. Tibiofemoral instability in primary total knee replacement: a review, Part 1: Basic principles and classification. Knee 2005;12:257-66.

24. Tokuhara Y, Kadoya Y, Nakagawa S, et al. The flexion gap in normal knees. An MRI study. J Bone Joint Surg Br 2004;86:1133-6.

25. Schipplein OD, Andriacchi TP. Interaction between active and passive knee stabilizers during level walking. J Orthop Res 1991;9:113-9.

26. Roth JD, Howell SM, Hull ML. Native Knee Laxities at $0^{\circ}$, $45^{\circ}$, and $90^{\circ}$ of Flexion and Their Relationship to the Goal of the Gap-Balancing Alignment Method of Total Knee Arthroplasty. J Bone Joint Surg Am 2015;97:1678-84.

27. Nowakowski AM, Majewski M, Müller-Gerbl M, et al. Measurement of knee joint gaps without bone resection: "physiologic" extension and flexion gaps in total knee arthroplasty are asymmetric and unequal and anterior and posterior cruciate ligament resections produce different gap changes. J Orthop Res 2012;30:522-7.

28. Tang H, Chen H, Yang D, et al. Distinctions of introarticular force distribution between genesis-II posterior stabilized and cruciate retaining total knee arthroplasty: An intraoperative comparative study of 45 patients. Clin Biomech (Bristol, Avon) 2017;42:1-8.

29. Laskin RS. The Insall Award. Total knee replacement with posterior cruciate ligament retention in patients with a fixed varus deformity. Clin Orthop Relat Res 1996;(331):29-34. 